\title{
Reconstruction of the Postsubiculum Head Direction Signal From Neural Ensembles
}

\author{
Adam Johnson, Kelsey Seeland, and A. David Redish
}

\begin{abstract}
Head direction cells change their firing rates as a function of the orientation of an animal within an environment. Typically, these cells display a unimodal tuning curve with maximal firing at the cell's preferred direction. As different cells have different preferred directions, the population of cells has been hypothesized to represent the orientation of the animal within the environment. Previous research has shown that pairs of simultaneously recorded head direction cells respond similarly to cue manipulations, suggesting that a population of head direction cells acts in concert to represent the animal's orientation within its environment. Ensembles of head direction cells were recorded from the postsubiculum from rats foraging in an open field. Directional responses of each cell were quantified by the nonparametric Watson's $U^{2}$ statistic, a measure which makes no explicit assumptions of tuning curve shape. Directionally responsive cells were then used to reconstruct each animal's orientation within the open field using population vector, optimal-linear estimator, and Bayesian methods. The results indicated that postsubiculum contained a complete representation of the animal's orientation. The internal consistency of a neural ensemble can be assessed by comparing the ensemble activity to the expected activity given the reconstructed orientation. This has been termed the "coherency" of the neural ensemble. Reconstruction error decreased as the coherency of the orientation representation increased, indicating that coherency could be used to measure a level of confidence in the representation quality. Because coherency is a linear measure dependent only on internal variables, coherency may be a behaviorally relevant measure used to ascertain the animal's confidence in its representation of orientation. ๑) 2004 Wiley-Liss, Inc.
\end{abstract}

KEY WORDS: neural ensemble; tetrode; rodent; spatial navigation; coherency

\section{INTRODUCTION}

Head direction cells respond to the orientation of an animal's head in the horizontal plane of its environment but show no dependence on the animal's location (Ranck, 1984). As defined by Taube et al. (1990a), each headdirection (HD) cell shows peak firing at a preferred direction and a monotonic fall-off as the orientation varies from that preferred direction. These cells have baseline-to-baseline tuning curve widths of $100^{\circ}$ (Taube et al., 1990a) and can be modeled as Gaussians with an average standard deviation

Department of Neuroscience, University of Minnesota, Minneapolis, Minnesota

Grant sponsor: National Institutes of Health; Grant number: NIH-R01MH68029-01; Grant sponsor: National Science Foundation; Grant number: NSF-IGERT9870633. Grant sponsor: Minnesota Medical Foundation. *Correspondence to: A. David Redish, Department of Neuroscience, 6-145 Jackson Hall, 321 Church St. SE, Minneapolis MN 55455.

E-mail: redish@ahc.umn.edu

Accepted for publication 21 May 2004

DOI 10.1002/hipo.20033

Published online 8 July 2004 in Wiley InterScience (www.interscience. wiley.com). of $66^{\circ}$ (Blair and Sharp, 1995). In rodents, HD cells have been found in postsubiculum (Taube et al., 1990a,b; Sharp, 1996), in the anterior dorsal thalamic nuclei (Blair and Sharp, 1995; Knierim et al., 1995; Taube, 1995; Blair et al., 1997), in the lateral mammillary nuclei (Blair et al., 1998; Stackman and Taube, 1998), as well as the dorsal tegmental nucleus of Gudden (Sharp et al., 2001b), the lateral dorsal nucleus of the thalamus (Mizumori and Williams, 1993), the striatum (Wiener, 1993; Ragozzino et al., 2001), and the retrosplenial cortex (Chen et al., 1994a,b).

HD cells were first detected in the postsubiculum (Ranck, 1984; Taube et al., 1990a). Controls showed that these tuning curves were independent of behavioral state and of the position of the animal and that the distribution of preferred directions was approximately uniform (Taube et al., 1990a). From 26\% (Taube et al., 1990 a) to $37 \%$ (Sharp, 1996) of postsubicular cells were found to be unimodal HD cells of the type defined by Taube et al. (1990a), however, Sharp (1996) reports that $89 \%$ of postsubicular cells showed a significant relationship between firing rate and the animal's head direction. Generally, HD cells have been recorded as animals foraged for food in a small cylinder ( $<1 \mathrm{~m}$ in diameter) with a cue card subtending about $90^{\circ}$ (Taube et al., 1990a,b; Taube, 1995; Knierim et al., 1995, 1998; Blair and Sharp, 1995, 1996; Cho and Sharp, 2001; Zugaro et al., 2003), but they have also been recorded in the standard eight-arm maze task (Chen et al., 1994a,b; Mizumori et al., 1999) and in more complex tasks in small environments (Wiener, 1993).

Rotations of external cues produce a corresponding rotation in the preferred directions of the recorded cells (Taube et al., 1990b). However, postsubicular HD cells continue to show normal tuning curves after removal of the cue card (Taube et al., 1990b) and in the dark (Taube et al., 1996). Postsubicular HD cells are dependent on anterior thalamic integrity (Goodridge and Taube, 1997) and anterior thalamic HD cells are dependent on lateral mammillary integrity (Blair et al., 1999). Postsubicular cells are also interconnected with the lateral dorsal nucleus (LDN) of the thalamus and with the retrosplenial cortex (van Groen and Wyss, 1990). In addition, postsubicular integrity is necessary for certain navigation tasks (Taube et al., 1992), and for the sensitivity of anterior thalamic head direction cells to cues (Goodridge and Taube, 1997). Animals with postsubicular lesions were significantly impaired at both the eightarm radial maze and the hidden-platform water maze 
(Taube et al., 1992). With postsubicular lesions, anterior thalamic cells continued to show HD tuning curves (although the tuning curves did broaden significantly), but they no longer followed cue card rotations (Goodridge and Taube, 1997). Anterior thalamic HD cells also showed more intrasession drift after postsubicular lesions than before (Goodridge and Taube, 1997). Thus, the postsubiculum is a critical component in the association between external cues and the internal head direction signal, and it should be possible to reconstruct the head direction of the animal from an ensemble of postsubicular HD cells.

Observations of head direction cell dynamics suggest HD cells act in concert and form a head direction system. Individual HD cells tend to show similar responses to manipulations (Taube et al., 1990b; Taube, 1995; Blair and Sharp, 1995). Studies in which pairs of HD cells have been simultaneously recorded (Taube et al., 1990b; Goodridge and Taube, 1995; Taube and Burton, 1995; Taube et al., 1996) have shown that pairs have responded similarly to environmental changes, suggesting that the set of HD cells work as an integrated system. Simultaneous recordings of HD and place cells have also suggested that they respond similarly to environmental manipulations (Knierim et al., 1998).

Research on head direction has benefited from a close interplay between theoretical models and experimental findings. Initial characterization of HD cells led to a model of the head direction system based on dead-reckoning or path integration of external and idiothetic cues (McNaughton et al., 1991). Attractor-based models of head direction (Skaggs et al., 1995; Redish et al., 1996; Zhang, 1996) were then developed in an effort to understand the dynamic properties of the head direction system. Attractor network models are composed of local excitatory and global inhibitory connections and represent head direction as a bump of activity with the cell population. These models are capable of producing realistic tuning curves while tracking realistic rotations (Redish et al., 1996; Redish, 1999; Goodridge and Touretzky, 2000; Sharp et al., 2001a).

Information encoded by HD cells suggest the presence of a complete orientation signal. If such a signal exists within a population, it should be possible to reconstruct the signal from the firing patterns within the population (Georgopoulos et al., 1983; Wilson and McNaughton, 1993; Rieke et al., 1997). Reconstruction has been used widely to test theories of population encoding in other biological systems (motor cortex: Georgopoulos et al., 1983; hippocampal place cells: Wilson and McNaughton, 1993; Zhang et al., 1998; Brown et al., 1998; Jensen and Lisman, 2000; LGN: Stanley et al., 1999). In the head direction system, it has only been used in models (Redish et al., 1996), because of the difficulty of recording an ensemble of HD cells. In this report we present the first neural ensembles of simultaneously recorded HD cells, and show that it is possible to reconstruct the rat head direction signal from those ensembles. Some of this work has been previously presented in abstract form (Johnson et al., 2003).

\section{MATERIALS AND METHODS}

\section{Experimental Subjects}

The subjects were two male, Brown-Norway/Fisher-344 hybrid rats (Harlan, Indianapolis IN). At the beginning of recordings, the rats were 13-15 months of age. During behavioral training and testing, the rats received their entire daily complement of food during the running session. The rats were weighed daily and maintained above $80 \%$ free-feed weight throughout the experiment. The rats were handled for $15 \mathrm{~min}$ each day. On days in which a rat foraged in the arena, the rats were handled after foraging. All procedures were approved by the IACUC at the University of Minnesota and were in accordance with National Institutes of Health (NIH) animal care guidelines.

\section{Task and Behavior}

The rats were trained to forage for food pellets in a black-walled cylindrical arena. The diameter of the arena was $91.5 \mathrm{~cm}$ and the height of the cylinder wall was $56 \mathrm{~cm}$. A white cue-card subtended $100^{\circ}$ of the arena wall; however, extensive distal cues were available outside the cylinder. Animals were brought into the experiment room inside their cage and placed in a terra cotta flower pot with towels for a 5-min pre-task recording. Rats were then placed near the center of the environment to begin the task. During the task, rats foraged for $20 \mathrm{~min}$ for 45-mg pellets (Research Diets, New Brunswick, NJ). Pellets were dropped randomly at a Poisson interval $(\lambda=10$-s mean) from three automatic food dispensers (MedAssociates, St. Albans, VT) placed at the top of the cylinder wall and separated by approximately $120^{\circ}$. Pellets generally covered the entire field. Following the task, the rats were immediately placed in the terra cotta flower pot for a 5-min post-task recording. The rats were trained for at least 2 weeks before surgery to ensure coverage of the entire open field. Following surgery and recovery, the animals were re-trained on the task until they reached pre-training activity levels. Rats were not explicitly disoriented at any time during training.

\section{Electrode Implantation}

The rats were implanted with 14-tetrode hyperdrives (David Kopf Instruments, Tujunga, CA). Twelve tetrodes were used to record neural activity, and two single-wire electrodes were used as references. Tetrodes were constructed from four lengths of $0.013-\mathrm{mm}$ wire insulated with polyamide (Kanthal Precision Wire, Palm Coast, FL). Rats were anesthetized with Nembutal (sodium pentobarbital, 40-50 mg/kg, Abbott Laboratories, North Chicago, IL) and the area of the implantation was shaved.

The rats were then placed on a stereotaxic apparatus (Kopf) and 0.1-ml Dual-cillin (Phoenix Pharmaceutical, St. Joseph, MI) was injected intramuscularly into each hindlimb. During surgery, anesthesia was maintained using isoflurane (vaporized to a level of $0.5-2 \%$ isoflurane in medical-grade oxygen). The scalp was then disinfected with alcohol and swabbed with Betadine (Purdue Frederick, Norwalk, CT). The skin overlying the skull was incised and retracted, and the underlying fascia was cleared from the surface of the skull. Excess bleeding was stopped by application of hydrogen peroxide followed by cautery of the retracted fascia. Anchor screws and one ground screw were placed in the skull, and a $1.8-\mathrm{mm}$ diameter craniotomy was opened using a surgical trephine (Fine Science Tools, Foster City, CA). The hyperdrive was positioned over right postsubiculum (bregma +7.0-mm AP, 2.0-2.4-mm 
$\mathrm{ML}$ ), and lowered to $1 \mathrm{~mm}$ below the surface of the skull. The craniotomy was protected using silastic (Dow Corning 3140) and the hyperdrive was secured in place with dental acrylic (Perm Reline and Repair Resin, Hygenic Corp., Akron, OH). Following surgery, $10 \mathrm{ml}$ sterile saline $(0.9 \%)$ was administered subcutaneously, and all tetrodes were advanced approximately $1 \mathrm{~mm}$. Animals were allowed to recover in an incubator until they were ambulatory, which was usually $1-2 \mathrm{~h}$ following surgery. Once the animals were ambulatory, $0.8 \mathrm{~mL}$ Children's Tylenol was administered orally. For 2 days following surgery, rats had access to water containing Children's Tylenol ( $25 \mathrm{ml}$ in $275 \mathrm{ml}$ of water). The rats were allowed 2 days to recover from surgery before resuming behavioral experiments.

\section{Neurophysiology}

Neural activity was recorded using a 54-channel Cheetah recording system (Neuralynx, Tucson, AZ). Voltage was sampled at $32 \mathrm{kHz}$ for each channel and filtered between $600 \mathrm{~Hz}$ and $6 \mathrm{kHz}$. When the voltage on any of the four channels of a single tetrode reached threshold, a 1-ms window of the spike waveform on each of the four channels on the tetrode was recorded and time-stamped with microsecond resolution (Neuralynx). Following the experiment, spikes were manually clustered into putative cells on the basis of their waveform properties (MClust 3.0; Redish and Schmitzer-Torbert, 2002), based on automatically-derived clusters found via k-means and expectation-maximization algorithms (KlustaKwik 1.0; Harris, 2002). Waveform properties used for clustering were energy, peak amplitude, valley amplitude, spike width, and principal components.

\section{Behavioral Data Collection}

A $12-\mathrm{cm}$ boom with a blue LED at the front and a red LED at the back was attached to the head stage during recording sessions. The position of each LED was recorded at $60 \mathrm{~Hz}$ via a video camera mounted $190 \mathrm{~cm}$ above the open field and standard videotracking software (Neuralynx). Video tracker samples were removed in which the view of one or both LEDs was obstructed or the light threshold was not reached. After each session, the HD was computed by finding a vector defined by the position of the blue LED relative to the red LED and taking the inverse tangent of the relative position to obtain an angle between $-180^{\circ}$ and $180^{\circ}$.

\section{Data Analysis}

Spike trains were binned using 2-ms bins and convolved with a Gaussian ( $\mathrm{SD}=1 \mathrm{~s}$ ) to obtain an estimation of continuous firing rate. Estimated continuous firing rate samples were then resampled at $60 \mathrm{~Hz}$ in order to temporally align these values with video tracker samples.

\section{Watson's $U^{2}$ test}

Watson's $U^{2}$-test was used to determine the extent to which a spike train encoded orientation information. Watson's $U^{2}$-test is a nonparametric test for data drawn from a circular distribution. Whereas most decoding measures require binning of the stimulus or response variable (e.g., orientation, spike data), Watson's $\mathrm{U}^{2}$ test compares distributions directly from observed samples. The null hypothesis is that the sample and test distributions are the same. The sample distribution was constructed by taking the rat's orientation at the time of each spike, and the test distribution was constructed by taking the orientation at each videotracker sample. It is important to note that Watson's statistical test does not have the same unimodal assumptions required by other standard parametric tests for circular data (e.g., Rayleigh tests) and, consequently, allows measurement of directional information from both unimodal and multimodal distributions (Zar, 1999).

Watson's $U^{2}$ effectively includes a measure of reliability in its measurement of orientation sensitivity. As can be seen in Figure 3, raw tuning curve shape is insufficient to determine whether orientation tuning is reliable across the session. A bootstrap (Efron, 1982) was used to visualize the reliability of observed tuning curves. For each cell, the orientation and orientation-at-time-ofspike distributions were randomly resampled 50 times at $20 \%$ density. A tuning curve was construced from each of these resamplings. Plotting the 50 bootstrap tuning curves allows visual inspection of the robustness of the actual tuning curve (see Fig. 3). An arbitrary cell selection threshold of $\mathrm{U}^{2} \geq 10$ was used to select "reliable" orientation sensitive cells.

A comparison of $\mathrm{U}^{2}$ and mutual information $\left(\mathrm{I}_{\mathrm{m}}\right)$ revealed that the log-transformed mutual information value was correlated with the $\log$ transformed $\mathrm{U}^{2}$ value $\left(\mathrm{r}^{2}=0.71\right)$. Furthermore, similar sets of cells were selected at thresholds of $U^{2} \geq 10$ and $I_{m} \geq 0.5$ bits. The calculation of mutual information, however, requires binning both stimulus and response variables; therefore, Watson's $\mathrm{U}^{2}$ was used to select orientation sensitive cells.

\section{Reconstruction}

\section{Vector reconstruction}

The population vector (Georgopoulos et al., 1983, also known as the vector-mean, Mardia, 1972; Zar, 1999), reconstructs orientation from neural populations via linear combinations of firingrate-weighted basis vectors, given by the preferred direction of each cell. The preferred direction, $\overrightarrow{\mathrm{L}}_{\mathrm{i}}$, is found as the weighted mean of the cell's firing rate over all possible directions. These vectors are then weighted by the cell's firing rate at time $t, f_{i}(t)$, to yield a reconstructed value at each time $t, \phi_{\text {est }}(t)$.

$$
\phi_{\text {est }}(\mathrm{t})=\operatorname{angle}\left[\sum_{\mathrm{i}} \mathrm{f}_{\mathrm{i}}(\mathrm{t}) \overrightarrow{\mathrm{L}}_{\mathrm{i}}\right]
$$

Behavioral and neural data were divided into two halves by splitting the session into interleaved 15 -s bins. Firing rates were selected from one half of the data and the basis vectors, $\overrightarrow{\mathrm{L}}_{\mathrm{i}}$, were calculated using tuning curves generated from the other half of the data.

\section{Optimal linear estimator}

The optimal linear estimator method (OLE) (Salinas and Abbott, 1994) calculates basis vectors such that it minimizes the error between the reconstructed and the actual vectors. Unlike popula- 

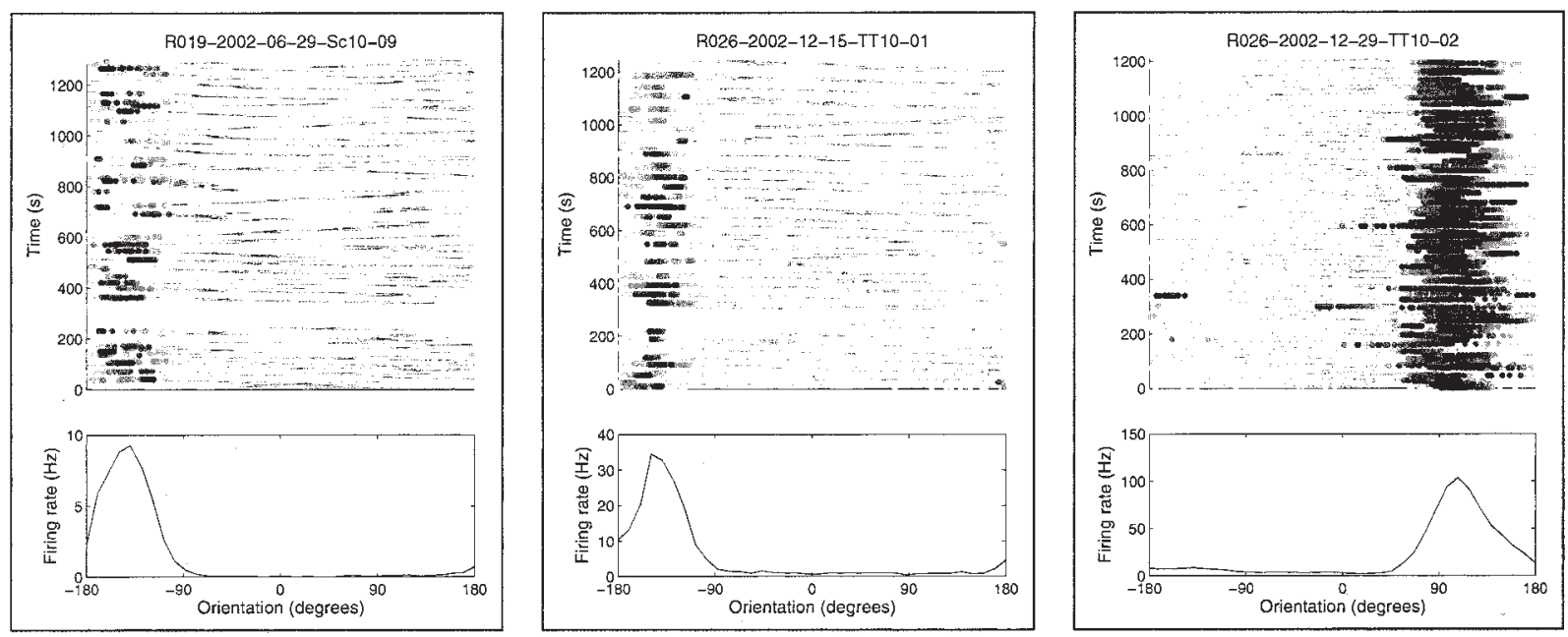

FIGURE 1. Examples of cells sensitive to orientation. Each dot in the top plot of in each panel represents the animal's head direction at each point in time. Each gray circle indicates the time and orientation of the animal when the cell fired a spike. Darkness of the circle indi- cates firing rate with darker circles indicating higher firing rates. (See Materials and Methods for details on estimation of instantaneous firing rate.) The $U^{2}$ values for each cell shown (from left to right) are $66.7,145.4$, and 557.3 , respectively. tion vector reconstruction, OLE does not require a uniform distribution of preferred directions. In OLE, correlations between tuning curves are exploited to remap the original basis vectors, given by preferred directions, to an optimum set of vectors, producing a new vector, $\vec{D}_{i}$, for each cell. These vectors are then weighted by the cell's firing rate at time $t, f_{i}(t)$, to yield a reconstructed value at each time $\mathrm{t}, \phi_{\text {est }}(\mathrm{t})$.

$$
\phi_{\text {est }}(\mathrm{t})=\operatorname{angle}\left[\sum_{\mathrm{i}} \mathrm{f}_{\mathrm{i}}(\mathrm{t}) \overrightarrow{\mathrm{D}}_{\mathrm{i}}\right]
$$

As with vector reconstruction, behavioral and neural data were divided into two halves by splitting the session into interleaved 15-s bins. Firing rates were selected from one half of the data and the basis vectors, $\vec{D}_{i}$, were calculated using tuning curves generated from the other half of the data.

\section{Bayes method}

The Bayesian method of reconstruction is a probability-based method of reconstruction using Bayes rule.

$$
\mathrm{P}(\phi \mid \mathbf{n})=\frac{\mathrm{P}(\mathbf{n} \mid \phi) \mathrm{P}(\phi)}{\mathrm{P}(\mathbf{n})}
$$

where $\mathbf{n}=\left(\mathrm{n}_{1}, \cdots, \mathrm{n}_{\mathrm{i}}\right)$ is the number of spikes fired by each cell during a given time interval, $\tau$. Following the single-step Bayesian reconstruction method outlined by Zhang et al. (1998), the probability distribution over $\phi$ given the activity of the ensemble, $\mathbf{n}$, can be computed. The reconstructed orientation, $\phi_{\text {est }}$, is given by the maximally likely orientation at each time point:

$$
\phi_{\text {est }}(\mathrm{t})=\arg _{\phi} \max [\mathrm{P}(\phi \mid \mathbf{n})]
$$

The method used (Zhang et al., 1998) assumes that the cells are Poisson and independent. Although neither of these assumptions are completely valid for our data, the method is not particularly sensitive to them, and Bayesian reconstruction was expected to reconstruct the animal's orientation reasonably well.

Again, behavioral and neural data were divided into two halves by splitting the session into interleaved 15 -s bins. The time interval $\tau$ was set to $1 \mathrm{~s}$. The probabilities and tuning curves were calculated using one-half of the data, then used to reconstruct the other half head direction from the ensemble neural activity, $\mathbf{n}$.

\section{Reconstruction accuracy-error angle}

Reconstruction accuracy was measured by comparing the reconstructed HD, $\phi_{\text {est }}(\mathrm{t})$, with the actual HD at time $\mathrm{t}, \phi(\mathrm{t})$, producing an error angle $\left(\mathrm{a}\right.$ value between $-180^{\circ}$ and $\left.180^{\circ}\right)$ :

$$
\mathrm{E}_{\phi}(\mathrm{t})=\phi(\mathrm{t})-\phi_{\text {est }}(\mathrm{t})
$$

Two measures were calculated for each session: median reconstructed error (median $\left(\mathrm{E}_{\phi}\right)$ ) and median absolute error (median $\left.\left(\left|\mathrm{E}_{\phi}\right|\right)\right)$. Median error provides a measure of the inherent bias in the reconstruction, while median absolute error provides a measure of the accuracy of the reconstruction.

\section{Representational quality-coherency}

Although reconstruction error measures the quality of reconstruction, it does not measure the quality of representation. For example, even random noise will yield a reconstructed $\mathrm{HD}$ value. An important question is thus to measure the quality of the representation. Redish et al. (2000) proposed measuring representation quality as the internal consistency of the ensemble, which they referred to as the coherency of the ensemble. Jackson and Redish (2003) presented a generalized coherency method that was applicable to any ensemble recording. Essentially, coherency measures the probability that the actual and expected activity of the system are the same. Mathematically, we calculate the actual and expected activity packets $\left(\mathrm{A}(\phi, \mathrm{t})\right.$ and $\mathrm{A}_{\mathrm{est}}(\phi, \mathrm{t})$, respectively) as the sum of 


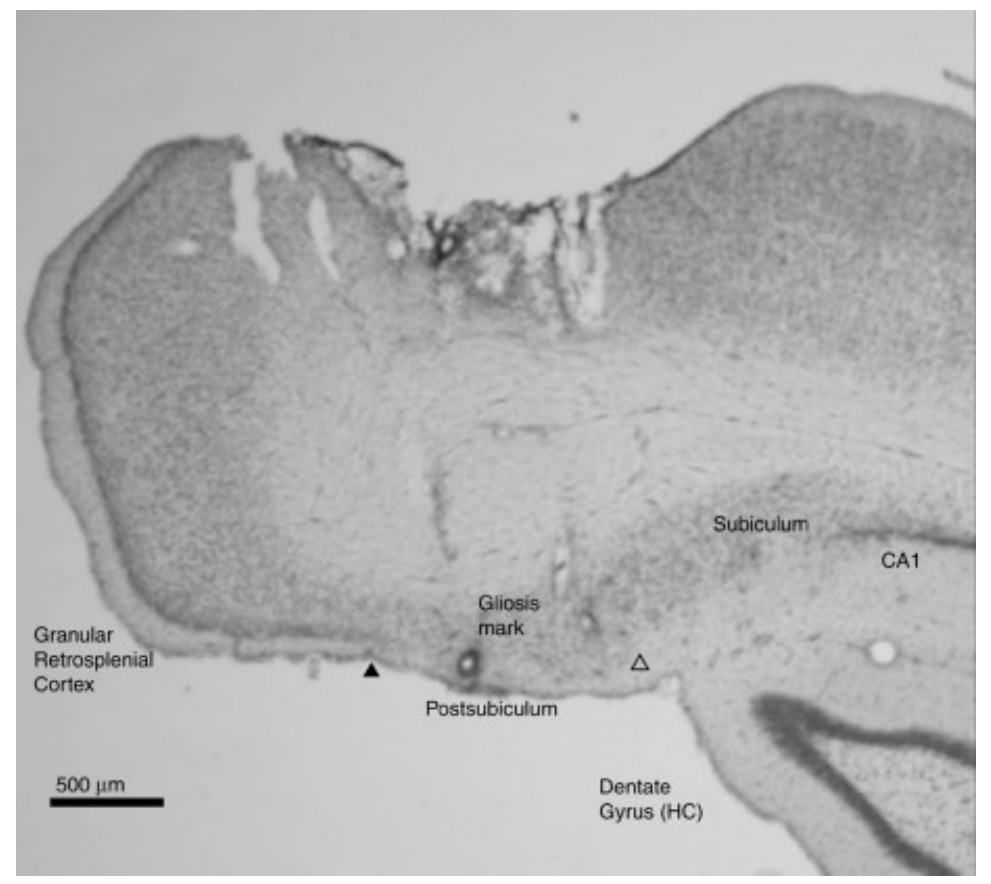

FIGURE 2. Cresyl violet stain of a coronal section showing tetrode gliosis mark in postsubiculum. The postsubiculum is bounded medially (filled triangle) by the retrosplenial cortex and laterally by the subiculum. This slice lies approximately 6-mm posterior to bregma. [R019 Tray-07 Slice-01].

the tuning curves of the cells, weighted by their firing rates. The actual activity packet, $\mathrm{A}(\phi, \mathrm{t})$, is based on the actual firing rates, while the expected activity packet, $A_{\text {est }}(\phi, t)$, is based on the expected firing rates, given the reconstructed orientation of the population at time $\mathrm{t}, \phi_{\text {est }}(\mathrm{t})$. Note that, like a tuning curve, the activity packet is a function over the HD variable $\phi$; unlike the tuning curve, however, the activity packet is also time dependent. Coherency is based on a comparison of the two activity packets (Redish et al., 2000) and was formally developed by Jackson and Redish (2003). In this formulation, an intermediate quantity called incoherency, I, quantifies the difference between activity packets. While Jackson and Redish (2003) calculated this quantity as the root-mean-squared error (RMSE) of the expected and actual activity packets, they also noted that incoherency can be calculated in a variety of ways. We calculated incoherency as

$$
I(t)=\frac{\operatorname{var}_{\phi}\left[A(\phi, t)-A_{\text {est }}(\phi, t)\right]}{\int_{\phi} A_{\text {est }}(\phi, t) d \phi}
$$

where $\operatorname{var}_{\phi}\left(\mathrm{A}(\phi, \mathrm{t})-\mathrm{A}_{\text {est }}(\phi, \mathrm{t})\right)$ is the variance of the difference between activity packets over $\phi$ at each time point $t$. Both the variance-based method and the RMSE-based method compare the shape of the activity packets, but the variance-based method has an added advantage because it is independent of baseline ensemble activity level.

From the incoherency measurement, we calculate the probability that the two curves are different through standard bootstrap techniques (Efron, 1982), producing a measurement between 0 (incoherent, i.e. unlikely that the actual packet was drawn from the same distribution as the expected) and 1 (coherent, i.e., likely that the actual and expect packets were drawn from the same distribution). Coherency values were found for each reconstructed HD sample. As in the reconstruction methods, data from a single session were halved using interleaved 15 -s bins. The incoherency distributions were obtained from one half and used to provide a probability estimate for the other half.

\section{HISTOLOGY}

Following the experiment, small lesions were made by passing current through the recording electrodes to identify recording sites $(5 \mu \mathrm{A}$ for $5 \mathrm{~s}$ ). After $24-48 \mathrm{~h}$, the rats were sacrificed by overdose of nembutal $(1.0 \mathrm{ml})$ and perfused intracardially first with saline, and then with $10 \%$ formalin. After the brains were removed, they were placed in $10 \%$ formalin overnight, then in a 30\% sucrose/ formalin mixture until they were sliced. Each brain was sliced using a freezing microtome in $40-\mu \mathrm{m}$ coronal sections, stored in formalin at $4^{\circ} \mathrm{C}$ until staining, and stained with cresyl violet.

\section{RESULTS}

A total of 390 spike trains were recorded from two rats over 17 recording sessions. Spike trains were identified as traditional head 

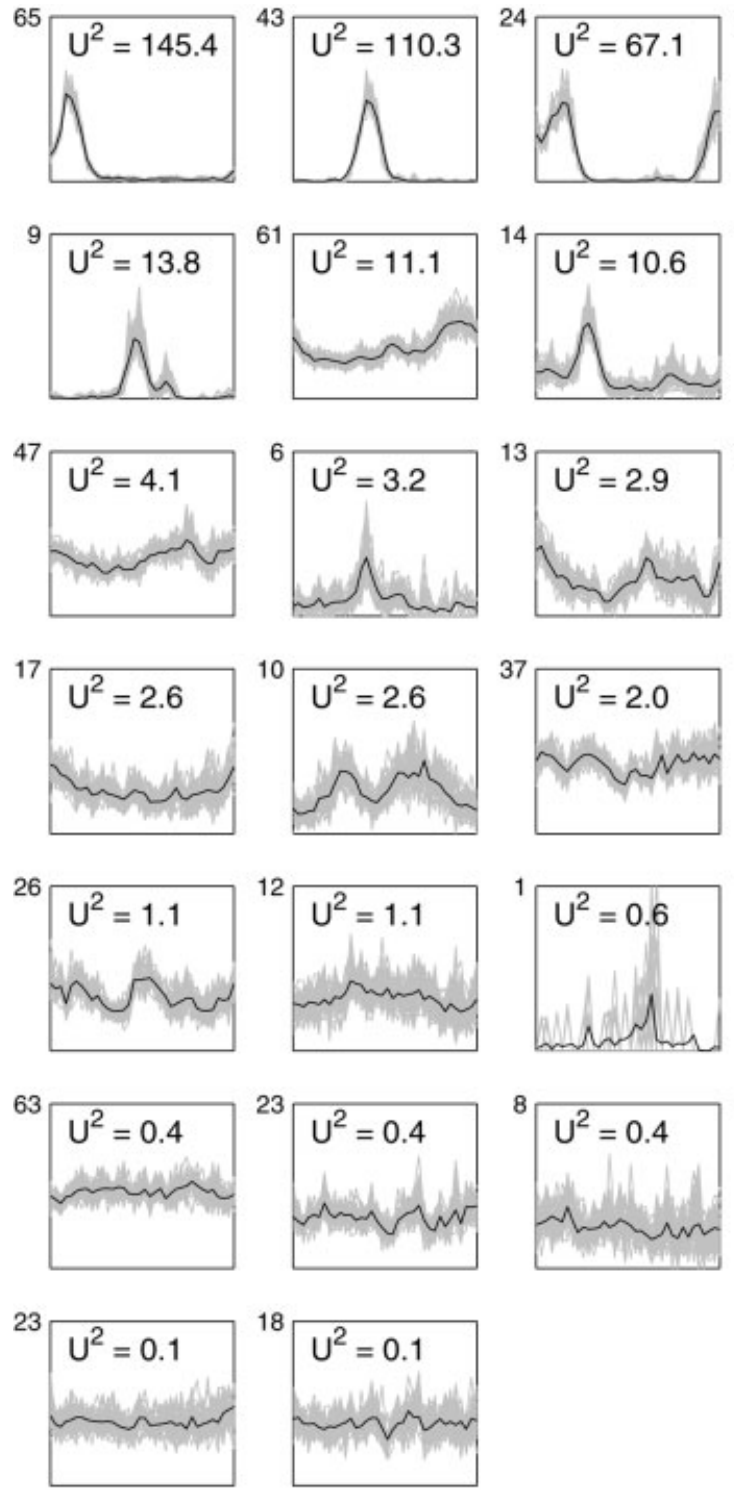

FIGURE 3. Head direction tuning curves for an ensemble of 38 simultaneously recorded cells. Each panel shows the tuning curve for one cell. The orientation of the animal is shown on the $\mathrm{x}$-axis $\left(-180^{\circ}\right.$ to $180^{\circ}$ ) and the firing rate on the $y$-axis (range $0 \mathrm{~Hz}$ to indicated value). Tuning curve of the cell is shown in black. The gray area

direction cells as defined by Taube et al. (1990a) $(\mathrm{n}=38$, visual inspection), cells sensitive to directional information ( $\mathrm{n}=45$, $\left.\mathrm{U}^{2} \geq 10\right)$, or cells insensitive to directional information $(\mathrm{n}=345$, $\left.\mathrm{U}^{2}<10\right)$. Figure 1 shows examples of typical HD cells.

\section{Histology}

Histology verified that tetrodes from the first rat (R019) were in postsubiculum (see Fig. 2); however, the location of tetrodes in the second rat (R026) could not be ascertained due to damage that occurred during brain extraction. Because cells recorded from the second rat (R026) were similar to those from the first rat, we assume that all tetrodes were placed in postsubiculum.
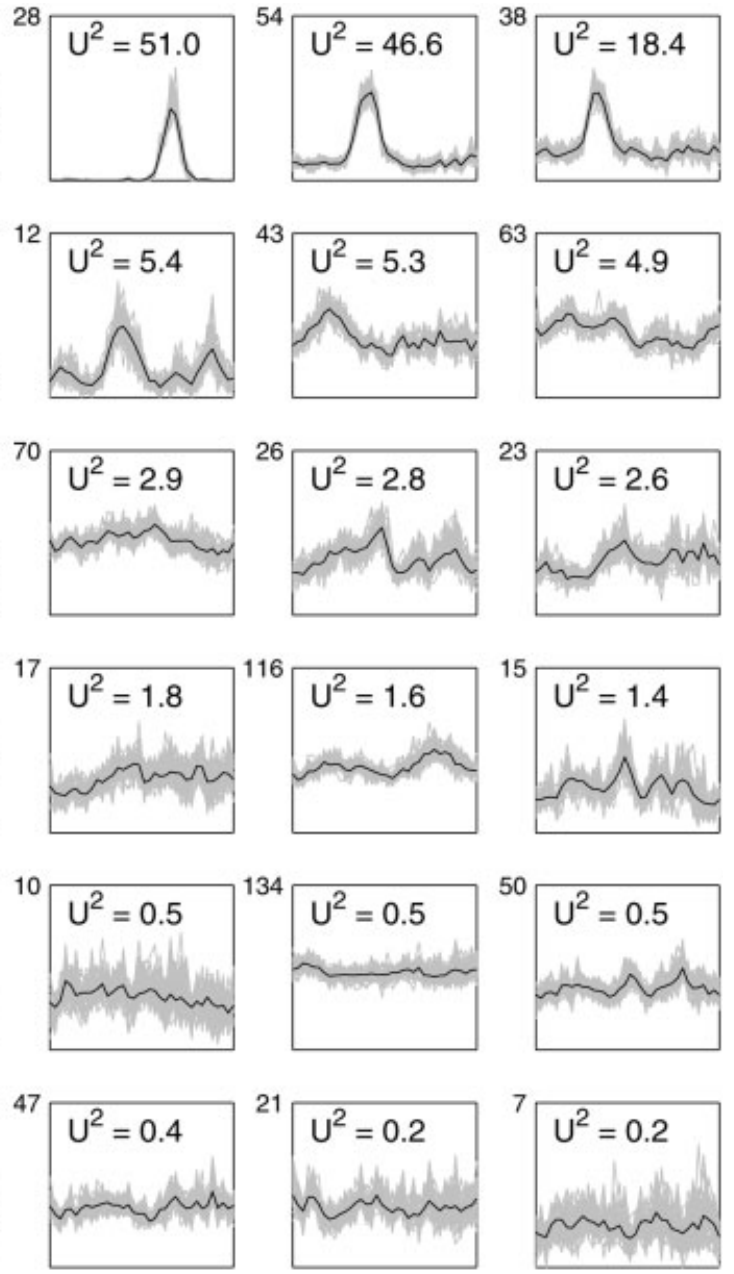

represents tuning curves derived from random resamplings of $20 \%$ of the spikes and orientation samples (see Materials and Methods for details). Fifty resamples were used. This provides for visualization of the reliability of the tuning curves. Session [R026-2002-12-15].

\section{Neural Ensembles of HD Cells}

Between 3 and 62 total spike trains were recorded in each session, producing up to nine HD-sensitive cells, and up to eight classical head direction cells. Figure 3 shows an example of HD tuning curves for an ensemble of 38 cells simultaneously recorded and sorted by decreasing $\mathrm{U}^{2}$ values. Table 1 provides a session-bysession report of the number of cells recorded for sessions in which more than three cells were recorded.

\section{Reconstruction}

Under conditions when environment cues are manipulated, individual HD cells typically display similar responses with similar 
TABLE 1.

Session-by-Session Totals of the Number of Cells Recorded, Number of Traditional Head Direction Cells (Taube et al., 1990a), and Number of Cells Used for Reconstruction for Sessions in Which Reconstruction Was Attempted*

\begin{tabular}{lcccc}
\hline Animal & Date & $\begin{array}{c}\text { Cells } \\
\text { used }\end{array}$ & $\begin{array}{c}\text { Traditional } \\
\text { HD cells }\end{array}$ & $\begin{array}{c}\text { Total cells } \\
\text { recorded }\end{array}$ \\
\hline R019 & $2002-06-27$ & 4 & 3 & 20 \\
& $2002-06-29$ & 6 & 5 & 27 \\
\multirow{3}{*}{ R026 } & $2002-07-03$ & 6 & 5 & 62 \\
& $2002-12-15$ & 9 & 8 & 38 \\
& $2002-12-29$ & 4 & 4 & 34 \\
\hline
\end{tabular}

*Cells identified as directional and used for reconstruction had values of $U^{2} \geq 10$. All traditional head direction cells had values of $U^{2} \geq 10$.

time courses (Taube et al., 1990b; Goodridge and Taube, 1995; Taube and Burton, 1995; Knierim et al., 1995, 1998; Blair and Sharp, 1995, 1996; Zugaro et al., 2003). When pairs have been simultaneously recorded, they have responded similarly (Taube et al., 1990b, 1996; Goodridge and Taube, 1995; Taube and Burton, 1995), and suggest that the population of cells act in concert. Tuning curves, such as those shown in Figure 1, suggest that vector-based reconstruction of HD should be possible. Non-unimodal tuning curves are not appropriate for vector-based reconstruction methods but are appropriate for probabalistic methods such as Bayesian reconstruction. While previous research clearly demonstrates that single and multiple head direction cells respond to changes in orientation, it does not address whether the system encodes orientation within the population. The central tenet of decoding measures such as reconstruction is to determine whether enough information exists within the population to form a general signal, in the present context, head direction. To analyze this question further, we compared the vector-based population vector reconstruction (Georgopoulos et al., 1983; Mardia, 1972) and OLE reconstruction (Salinas and Abbott, 1994), and the probabilitybased Bayesian reconstruction (Zhang et al., 1998).

\section{Reconstruction parameterization}

Tuning curves and single cell firing probability estimates used in vector- and probability-based reconstruction, respectively, were found by dividing the orientation space into $3610^{\circ}$ bins. As stated above, estimated continuous firing rates were found by binning the number of spikes into 2-ms time bins, then convolving these discrete values with a Gaussian with standard deviation of $1 \mathrm{~s}$. Selection of the orientation bin size and the standard deviation of the firing rate convolution Gaussian were found by parameterizing and selecting the combination of parameters that typically resulted in the smallest median reconstruction error over all five sessions. We tested binning orientations into $20^{\circ}, 10^{\circ}$, and $6^{\circ}$ bins and standard deviations for firing rate convolution from $0.5 s$ to $1.5 s$ in $0.1-s$ steps. The median reconstruction error changed little when the orientation bin size changed; in contrast, the median reconstruction error showed a marked decrease (improved) when the firing rate convolution Gaussian standard deviation was $0.8-1.2$ s. A mixed-factors analysis of variance (ANOVA) was used to determine whether the choice of parameters differentially affected each reconstruction method. A main effect was found for the reconstruction method $(\mathrm{F}(2,396)=131, P<0.05)$. As expected, median absolute reconstruction error for the population vector method was significantly larger than both OLE and Bayesian methods (post hoc HSD, $P<0.05$ ). No other differences or interactions were observed in parameterization due to reconstruction method $(P>0.05)$.
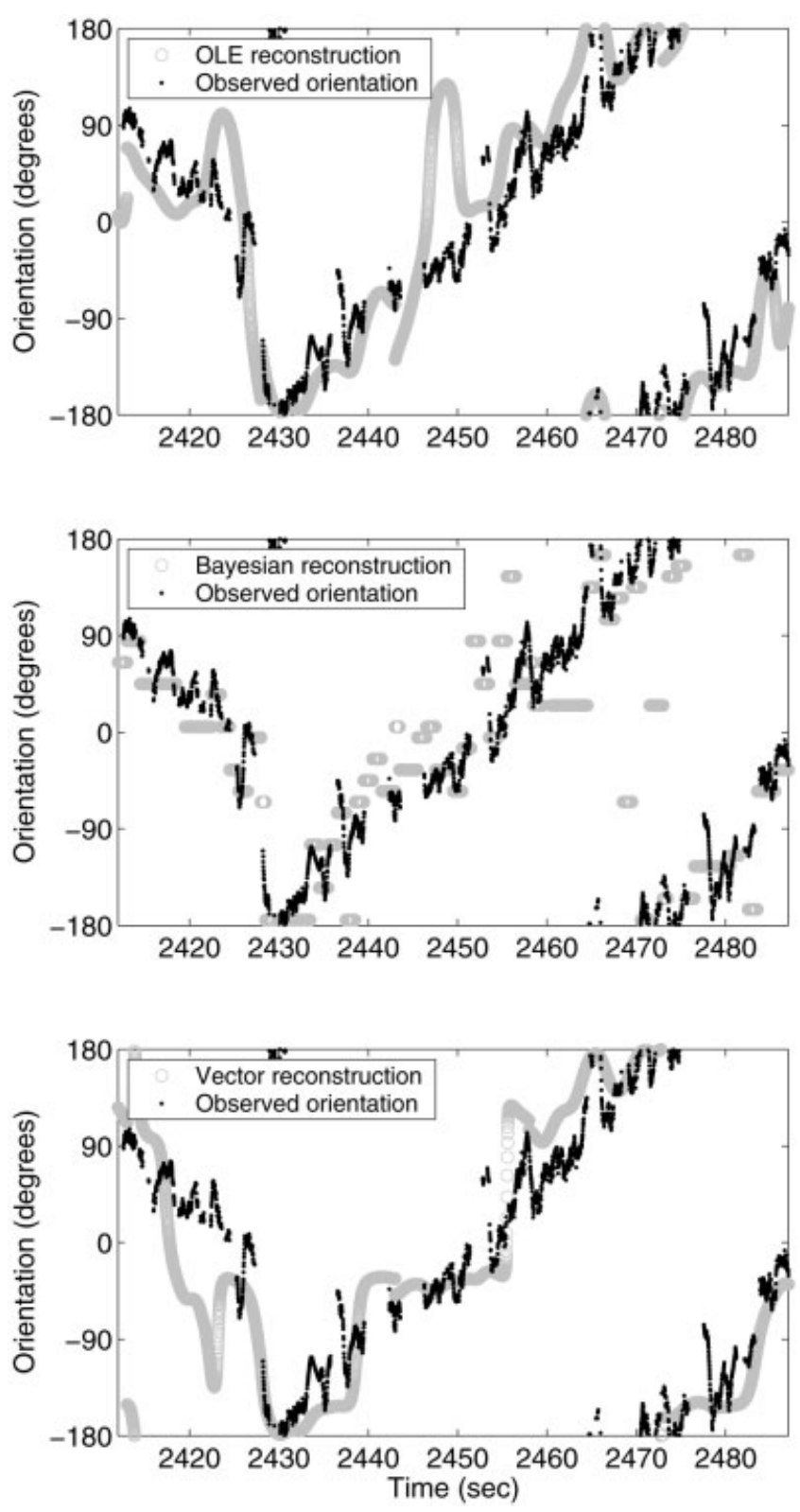

FIGURE 4. Reconstruction of head direction using OLE (top), Bayes (middle), and vector (bottom) reconstruction. Session [R0262002-12-15]. Data were halved using 15-s intervals. Nine cells met the threshold of $U^{2} \geq 10$ (see Materials and Methods) and were used for reconstruction. Gaps in the observed orientation resulted from an obstructed view of one or both of the tracking LEDs. 
TABLE 2

Session-by-Session Results for Reconstruction

\begin{tabular}{|c|c|c|c|c|c|c|c|}
\hline \multirow[b]{2}{*}{ Animal } & \multirow[b]{2}{*}{ Date } & \multicolumn{3}{|c|}{ Median absolute error } & \multicolumn{3}{|c|}{ Median error } \\
\hline & & OLE & Bayes & Vector & OLE & Bayes & Vector \\
\hline \multirow[t]{3}{*}{ R019 } & $2002-06-27$ & $40.5^{\circ}$ & $34.9^{\circ}$ & $67.5^{\circ}$ & $7.0^{\circ}$ & $6.6^{\circ}$ & $-46.7^{\circ}$ \\
\hline & $2002-06-29$ & $27.6^{\circ}$ & $29.6^{\circ}$ & $56.2^{\circ}$ & $-4.8^{\circ}$ & $-0.7^{\circ}$ & $3.6^{\circ}$ \\
\hline & $2002-07-03$ & $42.0^{\circ}$ & $48.3^{\circ}$ & $57.6^{\circ}$ & $-7.6^{\circ}$ & $15.9^{\circ}$ & $29.8^{\circ}$ \\
\hline \multirow[t]{2}{*}{ R026 } & $2002-12-15$ & $22.3^{\circ}$ & $18.4^{\circ}$ & $25.9^{\circ}$ & $-1.7^{\circ}$ & $0.1^{\circ}$ & $0.9^{\circ}$ \\
\hline & $2002-12-29$ & $24.3^{\circ}$ & $24.9^{\circ}$ & $50.0^{\circ}$ & $-3.4^{\circ}$ & $1.0^{\circ}$ & $11.5^{\circ}$ \\
\hline
\end{tabular}

The median absolute error provides an estimate of the reconstruction accuracy and the median error provides an estimate of the reconstruction bias. On average, Bayesian reconstruction performed best, OLE performed nearly as well, and standard vector reconstruction performed most poorly.

\section{Reconstruction results}

HD reconstruction was attempted for all sessions with three or more directionally sensitive cells. Figure 4 shows an example of reconstruction. Table 2 provides summary statistics of reconstruction for each session. Reconstruction using OLE and Bayes methods provided unbiased, accurate orientation estimates across sessions. Estimates of reconstruction bias were found by taking the median reconstruction error. Reconstruction using OLE and Bayes methods consistently showed little bias as both methods correct for asymmetric distributions of tuning curves. Because the distribution of tuning curves of the ensemble were often not uniformly distributed and population vector reconstruction makes no correction for this, the bias of population vector reconstruction varied greatly across sessions. Median absolute reconstruction error was used to assess reconstruction accuracy. Because only cells with unimodal tuning curves were used, absolute reconstruction error estimates for OLE and Bayes methods were similar within sessions. Like reconstruction bias, the accuracy of population vector based reconstruction displayed large session to session variability.

\section{Coherency}

Although reconstruction error measures the quality of reconstruction, it does not measure the quality of representation. Representational quality can be measured as the internal consistency of the neural ensemble (Redish et al., 2000; Jackson and Redish, 2003). By definition, high coherency values are associated with relative consistency within the ensemble and should produce, on average, good reconstruction. In contrast, low coherency values are associated with relative inconsistency within the ensemble and should produce reconstruction errors spread more randomly over all possible orientations.

Actual and expected activity packets were calculated as described in the Materials and Methods. Coherency was found for each reconstructed head direction sample. Linear regression was used to determine whether high coherency values were associated with relatively accurate reconstruction and low coherency values were associated with relatively inaccurate reconstruction within the ses- sion (Fig. 5). If the expected increasing coherency-decreasing error trend occurred, the slope of the fitted line should be negative, meaning that absolute reconstruction error decreased (improved) with increasing (improved) coherency. The expected coherencyerror trend was significant for each reconstruction method (OLE: $\mathrm{t}(4)=-2.67, P<0.05$; Bayes: $\mathrm{t}(4)=-3.12, P<0.05$; population vector: $\mathrm{t}(4)=-2.90, P<0.05$; one-tailed $t$-tests) (see Fig. 6). Coherency values and the coherency-error trend were dependent on the method of reconstruction and the activity of the ensemble (see Table 3). It should be noted that the coherency-error trend was maintained for population vector reconstruction because coherency is an indicator of relative reconstruction accuracy, not absolute reconstruction accuracy.

Coherency values and the coherency-error trend were dependent on the ensemble activity. For example, while one session ([R019-2002-06-27]) did not follow the increasing coherencydecreasing error trend, the ensemble consisted of cells with very low firing rates compared with other sessions. Because expected firing rates are based on mean firing rates, cells with low firing rates are poorly approximated, particularly when a cell sometimes fails to fire as the orientation passes through the cell's preferred direction. In this sense, finding the expected firing rate by tuning curves is inappropriate for cells with very low firing rates or cells that burst. Actual and expected activity packets, which are calculated by weighting tuning curves by firing rate and expected firing rate, respectively, are then no longer useful for calculating coherency.

\section{DISCUSSION}

The present results show the first recordings of HD cell ensembles, reconstruction of the head direction signal, and measurement of the representation quality in the postsubiculum head direction system. Moreover, the present results extend the findings of Jackson and Redish (2003) and demonstrate that coherency is a viable and useful measure of ensemble representation in biological systems, in that low representation quality predicted unreliable reconstruction. 

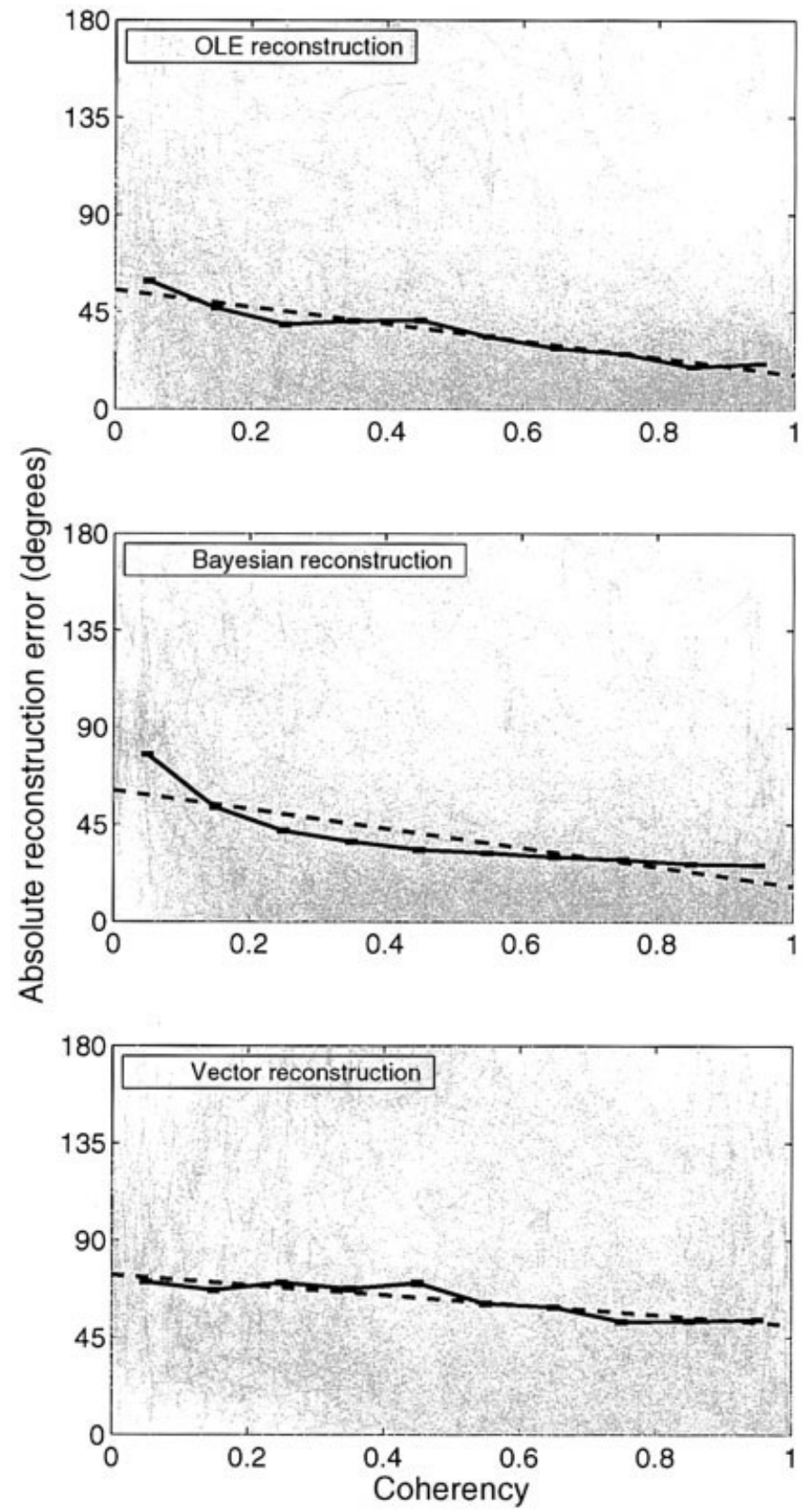

FIGURE 5. Absolute reconstruction error as a function of activity packet coherency (gray points) using OLE (top), Bayes (middle), and population vector (bottom) reconstruction for one session [R0262002-12-29]. The data were fit by a first-order polynomial (black dotted line) using least squares. The mean reconstruction error for each 0.1 coherency bin is shown by the solid black line.

\section{Tuning Curves}

HD cells are defined to have a unimodal tuning curve (Taube et al., 1990a), but postsubicular cells sensitive to head direction which do not fit this definition have also been observed (Sharp, 1996). Watson's $U^{2}$ test provides a useful quantitative measure of the directionality of cell response without explicit assumptions of tuning curve shape. Although cells with multimodal tuning curves were observed, these were typically low firing, and none met the selection criterion to be used for reconstruction.

\section{Implications of the Coherency Measurement}

Jackson and Redish (2003) showed that coherency was useful for detecting subtle changes in representation pertinent to different dynamic states of head direction models. In this paper, we found that coherency was a particularly useful measure of representational quality for biological investigations of head direction: incoherent ensembles generally showed poorer reconstruction than coherent ensembles. One interesting possibility is that an incoherent representation may suggest a behaviorally relevant state. Coherency as defined by Jackson and Redish (2003) is a linear measure. Thus it could be implemented with biologically plausible neurons, and may be exploited by the head direction system to determine the confidence of the animal's sense of HD.

\section{Implications for Modeling}

While theories of how HD cells maintain their correlations with orientation have converged on the idea that the system forms an attractor network (Skaggs et al., 1995; Redish et al., 1996; Zhang, 1996; Blair et al., 1998; Redish, 1999; Goodridge and Touretzky, 2000; Sharp et al., 2001a), the location of an attractor network is still under debate. Redish et al. (1996) suggested that attractor networks exist within both postsubiculum and the anterior dorsal thalamus. A later model developed by Goodridge and Touretzky (2000) placed the attractor network in postsubiculum, but updated through a lateral mammillary and anterior thalamic loop to

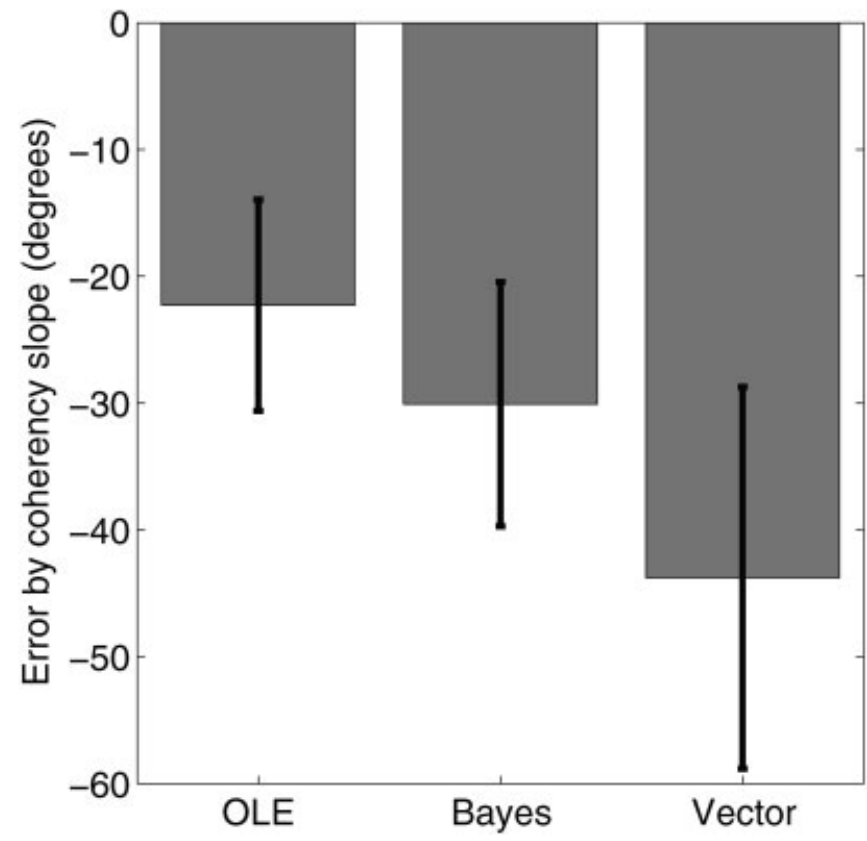

FIGURE 6. Mean slope of the absolute reconstruction error versus coherency for each reconstruction method. Error bars indicate the standard error of the mean. The slope of the fitted line was significantly less than zero for each reconstruction method (one-tailed $t$-test, $P<0.05)$. See Table 3 for session-by-session results. 
TABLE 3.

Session-by-Session Summaries of Reconstruction Error by Coherency Analysis*

\begin{tabular}{|c|c|c|c|c|c|c|}
\hline \multirow[b]{2}{*}{ Animal } & \multirow[b]{2}{*}{ Date } & \multicolumn{3}{|c|}{ Error by coherency slope $\left(^{\circ}\right)$} & \multirow{2}{*}{$\begin{array}{l}\text { Cells } \\
\text { used }\end{array}$} & \multirow{2}{*}{$\begin{array}{l}\text { Mean firing } \\
\text { rate }(\mathrm{Hz})\end{array}$} \\
\hline & & OLE & Bayes & Vector & & \\
\hline \multirow[t]{3}{*}{ R019 } & $2002-06-27$ & $8.7^{\circ}$ & $6.0^{\circ}$ & $-21.1^{\circ}$ & 4 & 2.22 \\
\hline & $2002-06-29$ & $-20.0^{\circ}$ & $-41.6^{\circ}$ & $-90.7^{\circ}$ & 6 & 13.80 \\
\hline & $2002-07-03$ & $-29.3^{\circ}$ & $-43.9^{\circ}$ & $-68.2^{\circ}$ & 6 & 6.11 \\
\hline \multirow[t]{2}{*}{ R026 } & $2002-12-15$ & $-31.4^{\circ}$ & $-26.1^{\circ}$ & $-15.6^{\circ}$ & 9 & 5.35 \\
\hline & $2002-12-29$ & $-39.4^{\circ}$ & $-44.7^{\circ}$ & $-23.2^{\circ}$ & 4 & 30.10 \\
\hline
\end{tabular}

*For each reconstructed orientation, the reconstruction error was plotted as a function of coherency. A line was fit to the data to obtain a slope. A negative slope indicates that as coherency increases reconstruction error decreases. The slope of the fitted line is listed for each reconstruction method.

reflect anatomical connectivity and tuning curve modulation. An alternative view presented by Blair and Sharp (Blair et al., 1997; Sharp et al., 2001a) suggested an interaction between the lateral mammillary nucleus and the dorsal tegmental nucleus as the locus of the attractor network. Despite these different perspectives, most theories assume that postsubiculum contains a complete HD signal (Taube et al., 1996; Redish, 1999; Sharp et al., 2001a). The data presented here confirm that postsubiculum does contain a complete HD signal. Lesion data showing that postsubicular tuning curves are dependent on anterior thalamic integrity (Goodridge and Taube, 1997) suggest that the postsubiculum may receive the HD signal from the anterior thalamus. However, it is not known whether the postsubiculum would continue to show a coherent representation (albeit not related to the orientation of the animal) after anterior thalamic lesions.

Models of HD predict the transformation of the HD signal between one area of the brain and another. Measures of decoding and representation quality are necessary to test how the signal is transformed and whether it is maintained as a unified signal at each point. Previous investigations of HD depended on average activity over many trials to obtain tuning curves. The present study used both decoding and representation quality measures to assess neural and behavioral correlations. These measures allow observation of dynamic processes over shorter time periods than tuning curves. Critical tests of head direction theories, particularly those based on attractor networks, depend on dynamics. Although the present study did not explicitly address dynamics, it demonstrates that decoding and representation quality measures will be useful in exploring the head direction system of the rat.

\section{Acknowledgments}

The authors thank Neil Schmitzer-Torbert and Jadin Jackson for helpful discussions and Deborah Bang for histological analysis. We also thank Jon Waataja, Mallika Arudi, Deborah Bang, Dan Bernal, Chris Boldt, Giuseppe Cortese, Monica Kumar, Susan Nwoke, and Tim Singewald for help running experiments. This work was supported by NIH-R01-MH68029-01. A.J. was partially supported by a 3M fellowship and by NSF-IGERT 9870633 .
Some equipment used for this project was bought through a donation by the Minnesota Medical Foundation.

\section{REFERENCES}

Blair HT, Sharp PE. 1995. Anticipatory head direction signals in anterior thalamus: evidence for a thalamocortical circuit that integrates angular head motion to compute head direction. J Neurosci 15:6260-6270.

Blair HT, Sharp PE. 1996. Visual and vestibular influences on headdirection cells in the anterior thalamus of the rat. Behav Neurosci 110:643-660.

Blair HT, Lipscomb BW, Sharp PE. 1997. Anticipatory time intervals of head-direction cells in the anterior thalamus of the rat, implications for path integration in the head-direction circuit. J Neurophysiol 78:145159.

Blair HT, Cho J, Sharp PE. 1998. Role of the lateral mammillary nucleus in the rat head direction circuit: a combined single unit recording and lesion study. Neuron 21:1387-1397.

Blair HT, Cho J, Sharp PE. 1999. The anterior thalamic head-direction signal is abolished by bilateral but not unilateral lesions of the lateral mammillary nucleus. J Neurosci 19:6673-6683.

Brown EN, Frank LM, Tang D, Quirk MC, Wilson MA. 1998. A statistical paradigm for neural spike train decoding applied to position prediction from ensemble firing patterns of rat hippocampal place cells. J Neurosci 18:7411-7425.

Chen LL, Lin LH, Green EJ, Barnes CA, McNaughton BL. 1994a. Headdirection cells in the rat posterior cortex. I. Anatomical distribution and behavioral modulation. Exp Brain Res 101:8-23.

Chen LL, Lin LH, Barnes CA, McNaughton BL. 1994b. Head-direction cells in the rat posterior cortex. II. Contributions of visual and ideothetic information to the directional firing. Exp Brain Res 101: 24-34.

Cho J, Sharp PE. 2001. Head direction, place, and movement correlates for cells in the rat retrosplenial cortex. Behav Neurosci 115:3-25.

Efron B. 1982. The jackknife, the bootstrap, and other resampling plans. Vol. 38. Regional Conference Series in Applied Mathematics. Philadelphia, PA: Society for Industrial and Applied Mathematics.

Georgopoulos AP, Caminiti R, Kalaska JF, Massey JT. 1983. Spatial coding of movement: a hypothesis concerning the coding of movement direction by motor cortical populations. Exp Brain Res Suppl: 327-336. 
Goodridge JP, Taube JS. 1995. Preferential use of the landmark navigational system by head direction cells in rats. Behav Neurosci 109:4961.

Goodridge JP, Taube JS. 1997. Interaction between the postsubiculum and anterior thalamus in the generation of head direction cell activity. J Neuroscience 17:9315-9330.

Goodridge JP, Touretzky DS. 2000. Modeling attractor deformation in the rodent head-direction system. J Neurophysiol 83:3402-3410.

Harris K. 2002. KLUSTAKWIK automatic cluster analysis, version 1.0. http://osiris.rutgers.edu/Buzsaki/software/.

Jackson JC, Redish AD. 2003. Detecting dynamical changes within a simulated neural ensemble using a measure of representational quality. Network Comput Neural Syst 14:629-645.

Jensen O, Lisman JE. 2000. Position reconstruction from an ensemble of hippocampal place cells: contribution of theta phase encoding. J Neurophysiol 83:2602-2609.

Johnson A, Seeland KD, Redish AD. 2003. Head-direction ensembles recorded from awake, behaving rats in an open field under cue-conflict situations. Soc Neurosci Abs 289.6.

Knierim JJ, Kudrimoti HS, McNaughton BL. 1995. Place cells, head direction cells, and the learning of landmark stability. J Neurosci 15: $1648-1659$.

Knierim JJ, Kudrimoti HS, McNaughton BL. 1998. Interactions between idiothetic cues and external landmarks in the control of place cells and head direction cells. J Neurophysiol 80:425-446.

Mardia KV. 1972. Statistics of directional data. New York: Academic Press.

McNaughton BL, Chen LL, Markus EJ. 1991. "Dead reckoning," landmark learning, and the sense of direction: a neurophysiological and computational hypothesis. J Cogn Neurosci 3:190-202.

Mizumori SJY, Williams JD. 1993. Directionally selective mnemonic properties of neurons in the lateral dorsal nucleus of the thalamus of rats. J Neurosci 13:4015-4028.

Ragozzino KE, Leutgeb S, Mizumori SJY. 2001. Dorsal striatal head direction and hippocampal place representations during spatial navigation. Exp Brain Res 139:372-376.

Ranck JB Jr. 1984. Head-direction cells in the deep cell layers of dorsal presubiculum in freely moving rats. Soc Neurosci Abs 10:599.

Redish AD. 1999. Beyond the cognitive map: from place cells to episodic memory. Cambridge, MA: MIT Press.

Redish AD, Elga AN, Touretzky DS. 1996. A coupled attractor model of the rodent head direction system. Network Comput Neural Syst 7:671-685.

Redish AD, Rosenzweig ES, Bohanick JD, McNaughton BL, Barnes CA. 2000. Dynamics of hippocampal ensemble realignment: time vs. space. J Neurosci 20:9289-9309.

Redish AD, Schmitzer-Torbert NC. 2002. MCLUST spike sorting toolbox, version 3.0. http://www.cbc.umn.edu/ redish/mclust/.

Rieke F, Warland D, de Ruyter van Steveninck R, Bialek W. 1997. Spikes. Cambridge, MA: MIT Press.

Salinas E, Abbott L. 1994. Vector reconstruction from firing rates. J Comput Neurosci 1:89-107.

Sharp PE. 1996. Multiple spatial/behavioral correlates for cells in the rat postsubiculum: multiple regression analysis and comparison to other hippocampal areas. Cereb Cortex 6:238-259.
Sharp PE, Blair HT, Cho J. 2001a. The anatomical and computational basis of the rat head-direction cell signal. Trends Neurosci 24:289294.

Sharp PE, Tinkelman A, Cho J. 2001b. Angular velocity and head direction signals recorded from the dorsal tegmental nucleus of gudden in the rat: implications for path integration in the head direction cell circuit. Behav Neurosci 115:571-588.

Skaggs WE, Knierim JJ, Kudrimoti HS, McNaughton BL. 1995. A model of the neural basis of the rat's sense of direction. In: Tesauro G, Touretzky DS, Leen TK, editors. Advances in neural information processing systems 7. Cambridge, MA: MIT Press, p 173-180.

Stackman RW, Taube JS. 1998. Firing properties of rat lateral mammillary single units: head direction, head pitch, and angular head velocity. J Neurosci 18:9020-9037.

Stanley GB, Li FF, Dan Y. 1999. Reconstruction of natural scenes from ensemble responses in the lateral geniculate nucleus. J Neurosci 19: $8036-8042$.

Taube JS. 1995. Head direction cells recorded in the anterior thalamic nuclei of freely moving rats. J Neurosci 15:1953-1971.

Taube JS, Burton HL. 1995. Head direction cell activity monitored in a novel environment and during a cue conflict situation. J Neurophysiol 74:1953-1971.

Taube JS, Muller RU, Ranck JB Jr. 1990a. Head direction cells recorded from the postsubiculum in freely moving rats. I. Description and quantitative analysis. J Neurosci 10:420-435.

Taube JS, Muller RU, Ranck JB Jr. 1990b. Head direction cells recorded from the postsubiculum in freely moving rats. II. Effects of environmental manipulations. J Neurosci 10:436-447.

Taube JS, Klesslak JP, Cotman CW. 1992. Lesions of the rat postsubiculum impair performance on spatial tasks. Behav Neural Biol 5:131143.

Taube JS, Goodridge JP, Golob EJ, Dudchenko PA, Stackman RW. 1996. Processing the head direction cell signal: a review and commentary. Brain Res Bull 40:477-486.

van Groen T, Wyss JM. 1990. The postsubicular cortex in the rat: characterization of the fourth region of the subicular cortex and its connections. Brain Res 529:165-177.

Wiener SI. 1993. Spatial and behavioral correlates of striatal neurons in rats performing a self-initiated navigation task. J Neurosci 13:38023817.

Wilson MA, McNaughton BL. 1993. Dynamics of the hippocampal ensemble code for space. Science 261:1055-1058.

Zar JH. 1999. Biostatistical analysis. 4th ed. Upper Saddle River, NJ: Prentice-Hall.

Zhang K. 1996. Representation of spatial orientation by the intrinsic dynamics of the head-direction cell ensemble: a theory. J Neurosci 16:2112-2126.

Zhang K, Ginzburg I, McNaughton BL, Sejnowski TJ. 1998. Interpreting neuronal population activity by reconstruction: unified framework with application to hippocampal place cells. J Neurophysiol 79:10171044.

Zugaro MB, Arleo A, Berthoz A, Wiener SI. 2003. Rapid spatial reorientation and head direction cells. J Neurosci 23:3478-3482. 